Check for updates

Cite this: RSC Adv., 2017, 7, 53104

Received 23rd July 2017

Accepted 5th November 2017

DOI: $10.1039 / c 7 r a 08132 d$

rsc.li/rsc-advances

\section{Uniform core-shell PPy@carbon microsphere composites with a tunable shell thickness: the synthesis and their excellent microwave absorption performances in the X-band}

\begin{abstract}
Jian Liu, ab Zhongzhu Wang, ${ }^{\text {d }}$ Sajid ur Rehmana and Hong Bi ${ }^{\text {DD }}$ *ac
Highly uniform core-shell polypyrroleacarbon microspheres (PPy@CM) have been successfully constructed by oxidation polymerization of pyrrole as the shell on the core of carbon microspheres. The thickness of the PPy shell can be easily controlled by modulating the weight ratio of pyrrole monomer and carbon microspheres. Hydrophilic carbon microspheres with abundant hydroxyl groups have a very strong affinity to the conjugated chains of PPy, which is responsible for the spontaneous formation of the uniform core-shell structure. Coating the PPy shell on the carbon core could increase the complex permittivity and dielectric loss due to an additional interfacial polarization. With a proper thickness of the PPy shells, the improved dielectric loss would be induced. The microwave absorbing properties of the PPyaCM powders were investigated by dispersing them in paraffin wax with $40 \mathrm{wt} \%$ powders in the frequency range of 2-18 GHz. The PPyaCM-0.6 $(0.6 \mathrm{~g} \mathrm{CM})$ sample exhibits the maximum reflection loss of $-38.1 \mathrm{~dB}$ at $11.6 \mathrm{GHz}$ and a bandwidth of less than $-10 \mathrm{~dB}$ covering 11.17 to $12.26 \mathrm{GHz}$ with the thickness of $3 \mathrm{~mm}$. In addition, the absorption peak sites and bandwidth of the PPyaCM composites can be easily modulated not only by the thickness of the absorbers but also of the PPy shell of the PPyaCM composite. Thus, the PPyaCM composites can be considered as a new type of promising lightweight microwave absorbers to satisfy the applications of microwave absorption in the $8-18 \mathrm{GHz}$ range.
\end{abstract}

\section{Introduction}

In recent years, microwave absorbing materials have gained increasing attention not only for their significant application in the stealth technology of military field but also for their effective frequency regulation on the excessive electromagnetic interference and electromagnetic pollution in civil field. ${ }^{1-3}$ Usually, traditional microwave absorbers comprising magnetic and dielectric loss materials are used in many areas to eliminate the electromagnetic wave pollution. ${ }^{\mathbf{4 - 6}}$ To satisfy the requirements of its practical applications, an excellent microwave absorbing material should be characterized with low thickness, lightweight, broad frequency, and strong absorption. ${ }^{7,8}$ It is widely accepted that some conventionally mixed composites could not be qualified as the next generation of microwave absorbing materials and a rational design on the nano-scaled

${ }^{a}$ College of Chemistry and Chemical Engineering, Anhui University, Hefei, 230601, P. R. China.E-mail: Bihong@ahu.edu.cn

${ }^{b}$ Department of Chemistry and Chemical Engineering, Hefei Normal University, Hefei 230601, P. R. China

${ }^{c}$ Key Laboratory of Opto-electronic Information Acquisition and Manipulation Ministry of Education, Anhui University, Hefei 230601, P. R. China

${ }^{d}$ School of Physics \& Materials Science, Anhui University, Hefei 230601, P. R. China heterostructures is always necessary to produce an enhanced performance., ${ }^{9,10}$ It is well-known that nano-structural engineering is beneficial to improve the microwave absorption property. ${ }^{11,12}$ A recent progress indicates that the uniform coreshell heterostructures could significantly contribute to the microwave absorption due to their interfacial polarization, confinement effect, and complementary behavior. ${ }^{\mathbf{1 3 , 1 4}}$ In particular, in the core-shell structure, the shells act as dielectric materials, which can create an electric polarization centre and significantly improve the dielectric loss and the impedance match. ${ }^{15}$ In addition, the ordered microstructure and the homogeneous chemical composition of the uniform core-shell nano-composites will be also helpful to the functional reproducibility and processability. ${ }^{16}$ Currently, the research on nanocomposites, composed of a magnetic core ( $\mathrm{Ni}, \mathrm{FeNi}, \mathrm{Co}$, etc.) and a dielectric shell ( $\mathrm{C}$, ZnO, polyaniline, etc.) in the nanometer size, is very active in the microwave-absorption field due to their excellent microwave-absorption performance. ${ }^{\mathbf{1 7}}$ Compared to a single-component type of microwave absorber, the core-shell structured nano-composites exhibit a much stronger reflection loss. As some examples, $\mathrm{Fe}_{3} \mathrm{O}_{4} @ \mathrm{C},{ }^{18} \mathrm{PBOPy} /$ $\mathrm{PPy} / \mathrm{Fe}_{3} \mathrm{O}_{4},{ }^{19} \mathrm{FeSn}_{2} /$ carbon $^{15} \mathrm{CoFe}_{2} \mathrm{O}_{4} / \mathrm{CNTs}^{20}{ }^{20} \mathrm{PPy} / \mathrm{Fe}_{3} \mathrm{O}_{4},{ }^{21}$ $\mathrm{NiS}_{2} @ \mathrm{MoS}_{2},{ }^{22}$ and $\mathrm{BF} / \mathrm{PANI}^{23}$ are being extensively developed. 
The C-based materials are always expected to be lightweight and cost-effective with a wide absorption bandwidth. In addition, the flexible substrates such as polymers can be rolled, bent, and folded to fit in a limited space where they are required. Thus, the polymers filled with the C-based materials have been developed to be used as excellent absorbing materials. ${ }^{24,25}$ Polypyrrole (PPy), as a typical conducting polymer, has become one of the most attractive polymers due to its high conductivity, low density, stability, ease of preparation and good environment stability. ${ }^{26-28}$ In previous studies, PPy was frequently employed as one of the effective components in the microwave absorbing composites, where PPy could not only serve as an organic binder and protection coating but also improve the dielectric loss and the impedance matching. ${ }^{29-32}$ Numerous approaches have been undertaken to increase the electrical conductivity by using PPy as the shells to further improve the microwave absorption properties. ${ }^{33-36}$ For example, Zhang et al. deposited the PPy layers on the surface of SiC nanowires with an extremely low concentration of the pyrrole monomers (only $10 \mathrm{wt} \%$ ) and the enhanced microwave absorption of this novel core-shell composite was confirmed to be linked to its structural characteristics. ${ }^{35}$ Qiao et al. reported the synthesis of the well-dispersed core-shell $\mathrm{Fe}_{3} \mathrm{O}_{4}$ @PPy microspheres with a tunable shell thickness of PPy (from 20 to $80 \mathrm{~nm}$ ), whose absorption was significantly improved as compared with that of bare $\mathrm{Fe}_{3} \mathrm{O}_{4}$ nanoparticles. ${ }^{27}$ Although it has been reported that inorganic particles could play the role of nucleation sites for $\mathrm{PPy},{ }^{36,37}$ the interaction between the nucleation sites and the pyrrole monomers was weak. Thus, independent PPy and core-shell composites were usually produced simultaneously during in situ polymerization or surface modification, which led to difficulty in controlling the thickness of the polymer shell..$^{38-40}$ Thus, the complete understanding of the microwave absorption mechanism of the uniform core-shell composites is required.

Herein, a new type of the polypyrrole@carbon microsphere (PPy@CM) composite with a prominent microwave absorption property was synthesized by in situ polymerization of carbon microsphere as the core and polypyrrole as the shell. Compared with other inorganic particles, carbon microspheres have much stronger affinity to pyrrole monomers, as carbon and pyrrole are homologous organic molecules, which can take part in an in situ intercalation polymerization to produce the polypyrrole/carbon composite. ${ }^{41}$ The uniform core-shell PPy@CM composite can be formed automatically without any assisted methods or techniques and the polymerization of independent PPy is also avoided effectively. The thickness of the PPy shells can be easily controlled by changing the amount of pyrrole monomers. In particular, the uniform core-shell PPy@CM composites exhibit significantly enhanced microwave absorption properties, which are superior to those of pristine $\mathrm{CM}$ and PPy. The characteristic advantages of the core-shell PPy@CM composites, such as easy preparation, strong absorption, wide responding range, and ultralow density (free of metal particles), could promise them a bright prospect as novel microwave absorbing materials.

\section{Experimental}

\section{Materials}

Pyrrole monomer (Shanghai Chemical Co.) was distilled under a reduced pressure. Chiral camphor sulfonic acid (CSA) and glucose were obtained from Aladdin Industrial Corporation. Ammonium persulfate (APS) was A. R. grade (Sinopharm Chemical Reagent Co.) and used as received.

\section{Preparing of hydrophilic carbon microspheres}

Glucose $(5.95 \mathrm{~g})$ was dissolved in deionized water $(60 \mathrm{~mL})$ to form a transparent solution, which was placed in an $80 \mathrm{~mL}$ Teflon-sealed autoclave and maintained at $180^{\circ} \mathrm{C}$ for $12 \mathrm{~h}$. After the reaction, the black products were centrifuged and washed with distilled water and ethanol for several times, and then dried at $-40{ }^{\circ} \mathrm{C}$ under vacuum for $12 \mathrm{~h}$. Finally, the black powders of hydrophilic carbon microspheres (CM) were obtained and their surfaces contained a large number of hydroxyl groups.

\section{Synthesis of PPy@CM composites}

As shown in Scheme 1, $1.61 \mathrm{~g}$ pyrrole, $0.93 \mathrm{~g}$ CSA, and $0.6 \mathrm{~g}$ carbon spheres were dispersed in $500 \mathrm{~mL}$ deionized water under magnetic stirring at $20-30{ }^{\circ} \mathrm{C}$ for $10 \mathrm{~min}$ to obtain a uniform (homogeneous) solution. Then, $500 \mathrm{~mL}$ aqueous solution containing $5.47 \mathrm{~g}$ of APS was added to the above mixture. The resulting solution was stirred for a while to ensure complete mixing; then, the reaction was maintained without agitation for $20 \mathrm{~h}$ at $30^{\circ} \mathrm{C}$. Finally, the products were washed with deionized water and ethanol until the filtrates became colorless and then dried under vacuum at $-40{ }^{\circ} \mathrm{C}$ for $10 \mathrm{~h}$ to obtain the PPy@CM-0.6 microspheres. Following this, we regulated the content of CM and the PPy@CM-1.0 (1.0 g CM) and PPy@CM-0.2 (0.2 $\mathrm{g}$ CM) samples were obtained. As a control, the PPy sample was prepared by the similar in situ polymerization procedure with the same amounts of pyrrole and CSA initiated by APS in deionized water.

\section{Characterization}

The morphologies of the samples were characterized by scanning electron microscopy (SEM, Hitachi S-4800). The Fourier transform infrared (FT-IR) spectra of the samples were recorded using a NEXUS-870 spectrometer and the powder X-ray diffraction (XRD) patterns were collected on a Japan Rigaku D/MAX-cA X-ray diffractometer equipped with a $\mathrm{Cu} \mathrm{K} \alpha$ radiation $\left(\lambda=1.5406 \AA\right.$ ) over the $2 \theta$ range of $5-80^{\circ}$. Thermogravimetric analysis (TGA) was performed on a SDT Q600 by heating the

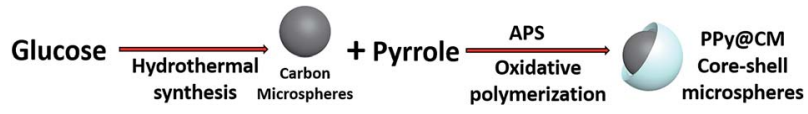

Scheme 1 Schematic illustration of the formation process of the core-shell PPyaCM- $x$ composite microspheres. 
samples at $10{ }^{\circ} \mathrm{C} \min ^{-1}$ from ambient temperature to $1000{ }^{\circ} \mathrm{C}$ in a $\mathrm{N}_{2}$ atmosphere.

\section{Electromagnetic parameters measurement}

The complex permeability and complex permittivity of the samples composing of paraffin wax and PPy@CM (mass ratio of $6: 4)$ were studied using an Agilent E5071C vector network analyzer in the frequency range from 2 to $18 \mathrm{GHz}$ at Anhui University. The as-prepared microwave absorbers were made into the toroidal-shaped specimens with $7.0 \mathrm{~mm}$ outer diameter and $3.04 \mathrm{~mm}$ inner diameter for the transmission/reflection measurements. The specimen was tightly inserted between the inner and outer conductor of the Agilent coaxial transmission airline for testing. The experimental reflection loss (RL) data of the toroidal-shaped specimens with different thicknesses $(1.5 \mathrm{~mm}, 2 \mathrm{~mm}, 2.5 \mathrm{~mm}, 3 \mathrm{~mm}, 3.5 \mathrm{~mm}, 4 \mathrm{~mm}, 4.5 \mathrm{~mm}$, and $5 \mathrm{~mm}$ ) were also collected using the terminated one-pot technique with a short $S_{11}$ test fixture.

\section{Results and discussion}

Fig. 1 shows the morphology evolution of CM (Fig. 1a), PPy (Fig. 1b), PPy@CM-1.0 (Fig. 1c), PPy@CM-0.6 (Fig. 1d and f), and PPy@CM-0.2 (Fig. 1e). The as-prepared CM shows the welldefined microspheres with a uniform microsphere diameter of about $700 \mathrm{~nm}$ (Fig. 1a), which is also determined by TEM image (Fig. 1a inset) and quite coincident with the previous report. ${ }^{42}$ The SEM image in Fig. 1b shows that the PPy sample has predominantly uniform nanospheres, which are assembled to form a coral-like shape. In the present condition, the change

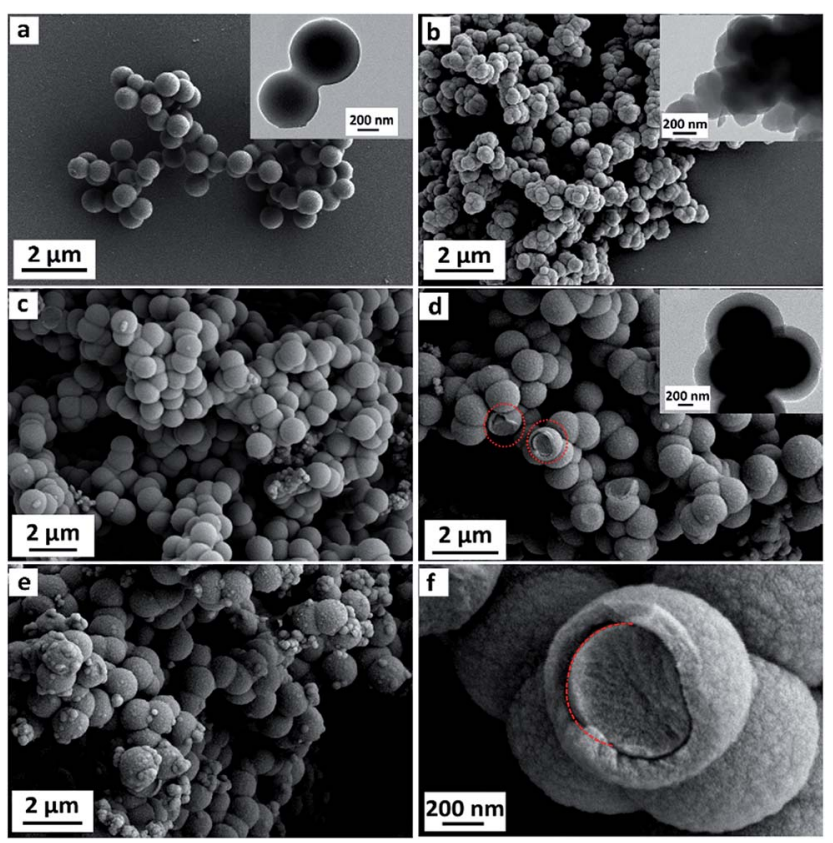

Fig. 1 SEM images of (a) CM (the inset is the TEM image of CM), (b) PPy (the inset is the TEM image of PPy), (c) PPyaCM-1.0, (d) PPyaCM-0.6 (the inset is the TEM image of PPy@CM-0.6), (e) PPy@CM-0.2, and ( $f$ ) high resolution SEM image of PPy@CM-0.6. in the morphology of PPy is primarily due to the doping acid in the water phase (Fig. 1b inset). Fig. 1c-e show the SEM images of the PPy@CM composites with different CM contents. These samples are similar in the morphology to pristine carbon microspheres. It is very interesting that the surface of the obtained composites becomes rough with some small bulges as compared with that of pristine carbon microspheres and their average diameters also present a monotonic increase when more pyrrole monomers are applied in the experiments, implying the formation of a new PPy shell. As representative images, Fig. 1d and $\mathrm{f}$ display some broken microspheres of PPy@CM-0.6, indicating the unique core-shell structures with an interior core and an outer shell. Notably, the highmagnification SEM image, as shown in Fig. 1f, presents that the interfaces between the CM core and the PPy shell are easy to be identified due to their different interphases and chemical compositions. In addition, these as-prepared composites possess highly uniform spherical microstructures and no impurities are detected until the mass ratio of CM to pyrrole monomers reaches $1: 8$, which demonstrates that this amount of pyrrole monomers is excessive and random polymerization cannot be restrained with further addition of pyrrole.

Based on the statistical data of diameter (Fig. 1c-e), the average diameters for PPy@CM-1.0, PPy@CM-0.6, and PPy@CM-0.2 are 0.98,1.14, and $1.18 \mu \mathrm{m}$, respectively. Since the diameter of the CM microsphere is about $700 \mathrm{~nm}$, the average shell thicknesses for these core-shell composites (PPy@CM-1.0, PPy@CM-0.6, and PPy@CM-0.2) are estimated to be about 140, 220 , and $240 \mathrm{~nm}$, respectively. These results indicate that, with a proper control of the experimental conditions and the relative ratios of pyrrole monomers to carbon microspheres, the PPy shells can be successfully fabricated on the CM cores and, in particular, the shell thickness is tunable at the nanoscale.

Fig. 2a shows the FT-IR spectra of CM, PPy, PPy@CM-1.0, PPy@CM-0.6, and PPy@CM-0.2 composites. For the PPy microspheres, some weak characteristic peaks of PPy, such as the $\mathrm{C}-\mathrm{N}$ stretching vibration in the ring $\left(1475 \mathrm{~cm}^{-1}\right)$, the $=\mathrm{C}-\mathrm{H}$ in-plane deformation modes $\left(1203 \mathrm{~cm}^{-1}\right)$, the $\mathrm{NH}^{+}$in-plane deformation vibration $\left(1110 \mathrm{~cm}^{-1}\right)$, the $\mathrm{C}-\mathrm{H}$ and $\mathrm{N}-\mathrm{H}$ inplane deformation vibrations $\left(1047 \mathrm{~cm}^{-1}\right)$, the $\mathrm{C}-\mathrm{C}$ out-ofplane ring deformation vibration $\left(925 \mathrm{~cm}^{-1}\right)$, and the $\mathrm{C}-\mathrm{H}$ out-of-plane deformation vibration of the ring $\left(797 \mathrm{~cm}^{-1}\right)$, can be observed, indicating the formation of PPy in the first step. ${ }^{\mathbf{4 3 , 4 4}}$ Similarly, all the characteristic peaks of PPy are also observed in the FT-IR spectra of the PPy@CM composites, further confirming that the new shells of PPy are formed on the surface of carbon microspheres. Even though the thickness of the PPy shells changes, the PPy@CM- $x$ composites with different contents of CM have shown the same characteristic peaks. By considering that the structure of the PPy shell is slightly different from the conventional PPy particles, it is reasonable to attribute the stronger affinity to the abundant surface groups of the carbon microspheres, which could lead to the formation of the substantial hydrogen bonds with the conjugated chains of PPy. This is the essential fact that carbon microspheres can stimulate the formation of the uniform core-shell PPy@CM- $x$ composites spontaneously. However, when more PPy is used in 

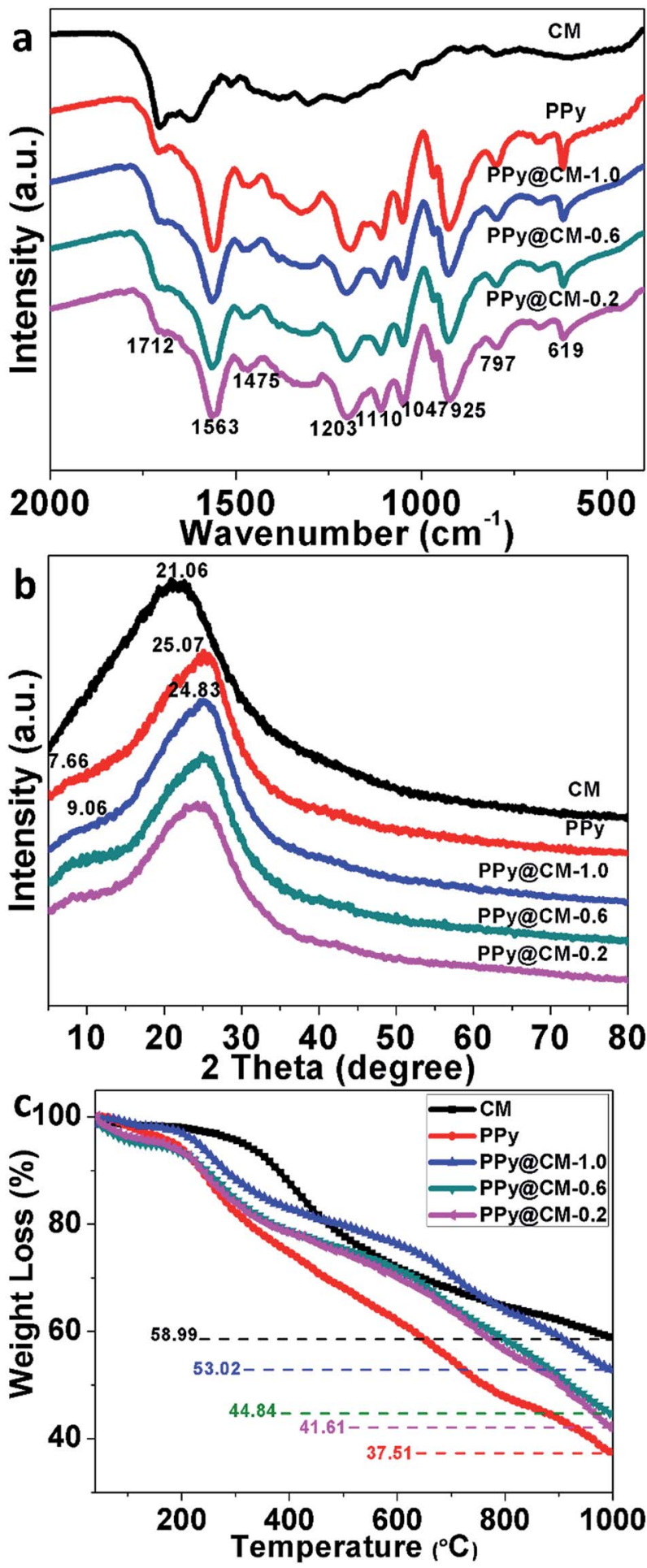

Fig. 2 (a) FT-IR spectra, (b) XRD patterns, and (c) TG curves of CM, PPy, PPy@CM-1.0, PPy@CM-0.6, and PPy@CM-0.2.

the PPy@CM- $x$ composites, the interaction between CM and the PPy shells will be gradually concealed and the composites will show the identical spectra to pristine PPy.

The XRD patterns of CM, PPy, PPy@CM-1.0, PPy@CM-0.6, and PPy@CM-0.2 are shown in Fig. 2b. The XRD patterns of the as-prepared CM and PPy reveal that both of them possess crystalline structure. The broad bread-shaped diffraction peak at $2 \theta=21.06^{\circ}$ in the XRD pattern of CM corresponds to the (002) lattice plane of a graphite phase. The broad peak at $2 \theta=25.07^{\circ}$ in the XRD pattern of PPy microspheres corresponds to the repetition units of regular pyrrole monomers. Moreover, due to the formation of the outer layer (i.e., the PPy shell), the XRD pattern of PPy@CM composites display a slightly shifted peak (24.83) compared with that of the PPy microspheres. However, the XRD diffraction peak of CM has not been detected in the asprepared PPy@CM- $x$ composites, which could be due to their PPy shell with relatively high thickness.

In view of the fact that PPy and CM are homologous organic materials, it is not easy to differentiate them exactly; therefore, the thermogravimetric analysis is utilized to estimate the content of PPy in these composites. According to the TG data, pyrrole monomer in the solution is fixed at $1.61 \mathrm{~g}$ and the final weight ratio for PPy@CM-1.0, PPy@CM-0.6, and PPy@CM-0.2 is 28.6\%, 56.9\%, and 58.1\%, respectively (Fig. 2c). It is observed that the total weights of PPy@CM-1.0 and PPy@CM-0.2 are significantly different from their theoretical outputs, which could result from the incomplete polymerization of pyrrole monomers and the loss of carbon microspheres. However, this situation would not occur in PPy@CM-0.6 due to the appropriate feed ratio of pyrrole monomers. Its yield can reach about $80 \%$, suggesting that the real composition in PPy@CM-0.6 composite is approximate to the designed values. ${ }^{45,46}$

Fig. 3 shows the complex permittivity $\left(\varepsilon^{\prime}\right.$ and $\left.\varepsilon^{\prime \prime}\right)$ and the complex permeability $\left(\mu^{\prime}\right.$ and $\left.\mu^{\prime \prime}\right)$ of the as-prepared powders embedded into wax ( $40 \mathrm{wt} \%$ powder) in the $2-18 \mathrm{GHz}$ range. As shown in Fig. 3a and b, for the pristine CM sample, the $\varepsilon^{\prime}$ value changes around 3.27 and the $\varepsilon^{\prime \prime}$ value increases from 0.04 to 0.27 with the frequency increase. For the pristine PPy sample, the $\varepsilon^{\prime}$ value changes between 6.2 and 4.09 and exhibits a slight peak with a value of 4.65 at $12.08 \mathrm{GHz}$. The $\varepsilon^{\prime \prime}$ value changes from 2.07 to 0.59 and exhibits a peak with a value of 0.99 at $13.92 \mathrm{GHz}$.
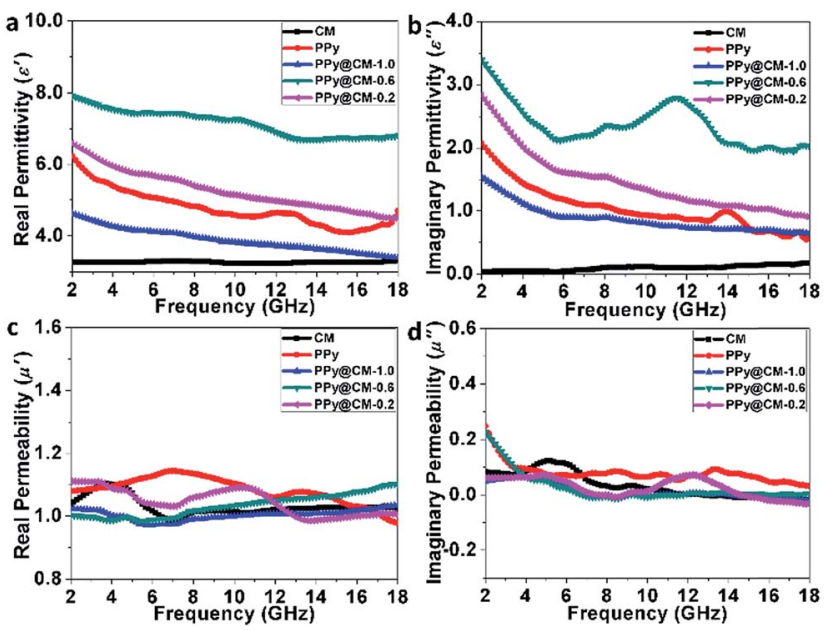

Fig. 3 (a) Real permittivity, (b) imaginary permittivity, (c) real permeability and (d) imaginary permeability of CM, PPy, PPy@CM-1.0, PPy@CM-0.6, and PPy@CM-0.2. 
For the PPy@CM- $x$ samples, as the CM content increases from $0.2 \mathrm{~g}$ to $1.0 \mathrm{~g}$, both $\varepsilon^{\prime}$ and $\varepsilon^{\prime \prime}$ first increase and then decrease. $\varepsilon^{\prime}$ of the PPy@CM-0.6 sample decreases from 7.91 to 6.67 with the increase in frequency, while its $\varepsilon^{\prime \prime}$ first decreases from 3.42 to 2.12 in the range of $2-5.84 \mathrm{GHz}$ and then presents a broad peak with the value of 2.79 at $11.52 \mathrm{GHz}$. Compared to other samples, the PPy@CM-0.6 sample exhibits a much higher complex permittivity and significantly high $\varepsilon^{\prime \prime}$ value (11.52 $\mathrm{GHz}$ ), which could be ascribed to the moderate occurrence of the stronger interfacial polarization between the CM core and the PPy shell. This indicates that the introduction of the PPy shell on the core of carbon microspheres can significantly enhance the dielectric loss as compared to that of pristine CM and pristine PPy. The enhanced dielectric loss can be ascribed to the synergistic effect, which has been reported previously. ${ }^{47}$ Moreover, the dielectric properties of the core-shell composite are much better than those of the physically combined CM and $\mathrm{PPy}^{48}$ In addition, the plots in Fig. $3 \mathrm{c}$ and $\mathrm{d}$ indicate that these samples are non-magnetic; thus, their magnetic loss can be neglected.

The dielectric dissipation factor $\left(\tan \delta_{\varepsilon}=\varepsilon^{\prime \prime} / \varepsilon^{\prime}\right)$ is a common concept to evaluate the dielectric loss rates of microwave absorbers. ${ }^{49,50}$ As shown in Fig. 4, both CM and PPy show quite low $\tan \delta_{\varepsilon}$ in the entire frequency range due to their small $\varepsilon^{\prime \prime}$ values. With the formation of the PPy shells on the surface of the carbon core, $\tan \delta_{\varepsilon}$ of the PPy@CM- $x$ composite can be substantially enhanced than that of pristine CM and PPy. However, as the CM content increases from $0.2 \mathrm{~g}$ to $1.0 \mathrm{~g}, \tan \delta_{\varepsilon}$ of the PPy@CM- $x$ composite first increases and then decreases. The PPy@CM-0.6 composite has the highest tan $\delta_{\varepsilon}$ value among those of other samples, which implies that the interfacial polarization would become the dominant mechanism for the dielectric loss once the thickness of the PPy shells reaches a critical value. It is widely accepted that the dielectric behavior of the permittivity in the microwave frequency range should primarily originate from the dipole polarization and the interface polarization. ${ }^{51,52}$ For the PPy@CM- $x$ composite, besides the intrinsic electric dipole polarization of the individual PPy shell

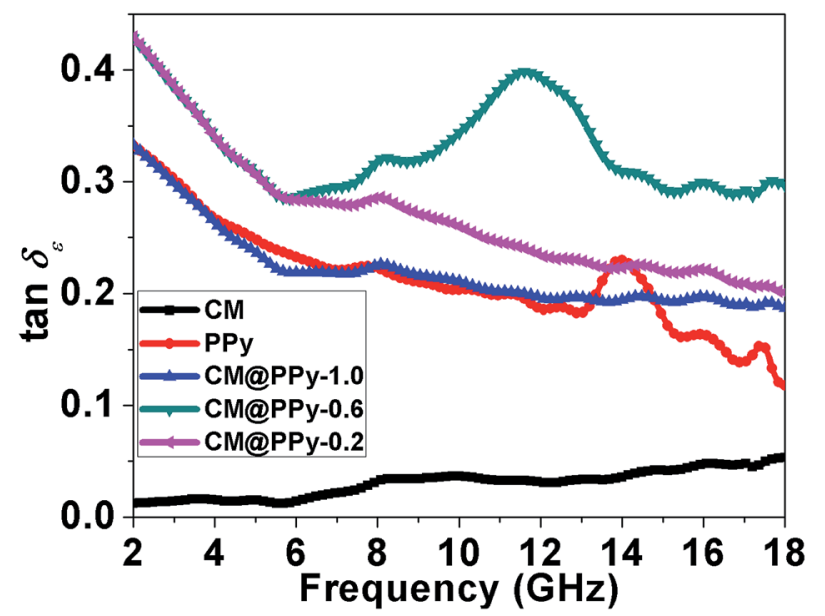

Fig. 4 Dielectric dissipation factors $\left(\tan \delta_{\varepsilon}\right)$ of CM, PPy, PPy@CM-1.0, PPy@CM-0.6 and PPy@CM-0.2. and the CM core, the interface polarization between them is dominant. In addition, according to the Maxwell-Garnet theory (MGT), ${ }^{53}$ the complex permittivity arising from the contribution of the interface polarization between the PPy shell and the CM core is in close relation with the electric conductance and the volume filling factor of the PPy shell. ${ }^{54}$ Thus, the interfacial polarization will become the dominant mechanism for the dielectric loss of the PPy@CM- $x$ composite once the thickness of PPy shells reaches a critical value. Clearly, the change of shell thickness should modulate the dielectric loss of the PPy@CM- $x$ composite.

The measured RL data of CM, PPy, PPy@CM-1.0, PPy@CM0.6, and PPy@CM-0.2 composite with a weight fraction of $40 \%$ in the paraffin matrix with different layer thicknesses are displayed in Fig. 5a-e. The maximum measured RL of CM is $-16.57 \mathrm{~dB}$ at $10.56 \mathrm{GHz}$ with the sample thickness of $3 \mathrm{~mm}$ and PPy has the maximum RL of $21.1 \mathrm{~dB}$ at $10.6 \mathrm{GHz}$ with the sample thickness of $3 \mathrm{~mm}$. In comparison with pristine $\mathrm{CM}$ and bare PPy particles, the PPy@CM- $x$ composite samples have the enhanced RL data (as shown in Fig. 5c-e), which can be explained as follows: first, the impedance match can be improved after a carbon microsphere is coated with the PPy shell; second, the strong effect of the PPy dielectric loss was helpful for microwave absorption; third, the interfacial polarization between the carbon core and the PPy shell was also favorable for the microwave absorption. In addition, among these PPy@CM- $x$ samples, the PPy@CM-0.6 sample exhibits the best microwave absorption performance. As shown in Fig. $5 \mathrm{~d}$, at
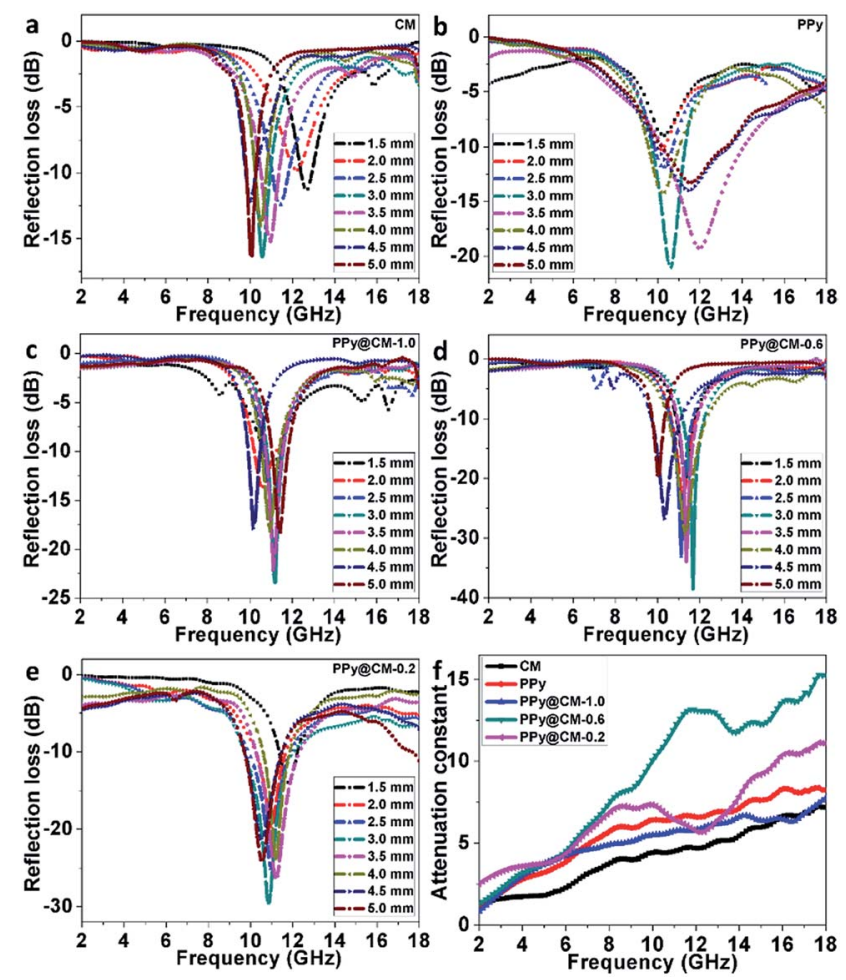

Fig. 5 (a-e) Measured reflection losses of the samples with different thicknesses (1.5-5.0 mm) and (f) attenuation constant of CM, PPy, PPy@CM-1.0, PPy@CM-0.6, and PPy@CM-0.2. 
the sample thickness of $3 \mathrm{~mm}$, the maximum RL of PPy@CM0.6 is $-38.1 \mathrm{~dB}$ at $11.6 \mathrm{GHz}$ and the bandwidth (below -10 dB) covers 11.17-12.26 GHz. The PPy@CM-0.6 sample exhibits the best electromagnetic absorption, which could be ascribed to the strongest dielectric loss.

Further, according to the transmission line theory, good absorbers have also high electromagnetic attenuation. The microwave attenuation is controlled by the attenuation constant $\alpha$, which can be described as: $:^{55,56}$

$$
\alpha=\frac{\sqrt{2} \pi f}{c} \times \sqrt{\left(\mu^{\prime \prime} \varepsilon^{\prime \prime}-\mu^{\prime} \varepsilon^{\prime}\right)+\sqrt{\left(\mu^{\prime \prime} \varepsilon^{\prime \prime}-\mu^{\prime} \varepsilon^{\prime}\right)^{2}+\left(\mu^{\prime} \varepsilon^{\prime \prime}+\mu^{\prime \prime} \varepsilon^{\prime}\right)^{2}}}
$$

where $f$ is the frequency of the EM-wave and $c$ is the velocity of light. Fig. $5 \mathrm{f}$ shows the frequency dependence of the attenuation constant. The PPy@CM-0.6 sample endows the highest $\alpha$ in all frequency ranges, indicating an excellent EM wave attenuation. From the above equation, it is noted that the higher imaginary parts of the permittivity and permeability appear to be useful for improving the attenuation constant. The enhancement of the microwave absorption properties of the PPy@CM-0.6 composite results from the increase in the dielectric loss. In our present case, PPy@CM-0.6 has potential applications as superior absorbents due to its lightweight and higher imaginary parts of the permittivity.

\section{Conclusions}

With carbon microspheres as the core and nucleation sites, the core-shell PPy@CM- $x$ composites have been successfully constructed through an in situ polymerization of pyrrole monomers. Since the hydroxyl groups are rather abundant on the surface of hydrophilic carbon microspheres, they can display a strong affinity to the conjugated chains of PPy through hydrogen bonding, resulting in the spontaneous formation of the highly uniform core-shell microstructure without the requirement of any pretreatment techniques. The thickness of the PPy shells can be well-controlled from 140 to $240 \mathrm{~nm}$ by changing the weight ratio of carbon microspheres and pyrrole monomer. In the core-shell structured composites, the additional interfaces can induce the interfacial polarization, which further contributes to the dielectric loss. Compared to pristine carbon microspheres and bare PPy particles, the PPy@CM-0.6 composite shows substantially enhanced microwave absorption properties with the maximum $\mathrm{RL}$ of $-38.1 \mathrm{~dB}$ at $11.6 \mathrm{GHz}$ and the bandwidth covering $11.17-12.26 \mathrm{GHz}$ at the sample thickness of $3 \mathrm{~mm}$. Its excellent microwave absorption performances could be ascribed to the high dielectric loss arising from the strong interface polarization between the CM core and the PPy shell. The microwave absorption performances of the as-prepared PPy@CM-0.6 composite are even better than those of numerous PPy-based composites ever reported. In particular, the microwave absorption properties of the as-prepared PPy@CM- $x$ composites are highly dependent on the thickness of the PPy shells, which indicates that the microwave absorption can be simply modulated not only by manipulating the absorber thickness but also by tuning the shell thickness to satisfy the applications in different frequency bands. Our results demonstrate that the PPy@CM- $x$ core-shell microspheres prepared in the current study are fascinating candidates for new types of microwave absorbing materials, which are metal-free, thin and exhibit strong absorption. This study would be helpful for the design and the development of some novel lightweight microwave absorbers with a uniform microstructure and an enhanced functional property.

\section{Conflicts of interest}

There are no conflicts to declare.

\section{Acknowledgements}

This work was financed by the National Natural Science Foundations of China (Grant no. 51272002), the University Science Research Project of Anhui Province (No. KJ2016A577) and the Anhui Provincial Natural Science Fund (No. 1708085ME123). All of the co-authors thank the Key Laboratory of EnvironmentFriendly Polymer Materials of Anhui Province and the Key Laboratory of Opto-electronic Information Acquisition and Manipulation, Ministry of Education, Anhui University.

\section{References}

1 Q. He, T. Yuan, X. Zhang, X. Yan, J. Guo, D. Ding, M. A. Khan, D. P. Young, A. Khasanov, Z. Luo, J. Liu, T. D. Shen, X. Liu, S. Wei and Z. Guo, J. Phys. Chem. C, 2014, 118, 24784-24796.

2 H. Sun, R. Che, X. You, Y. Jiang, Z. Yang, J. Deng, L. Qiu and H. Peng, Adv. Mater., 2014, 26, 8120-8125.

3 Y. C. Du, T. Liu, B. Yu, H. B. Gao, P. Xu, J. Y. Wang, X. H. Wang and X. J. Han, Mater. Chem. Phys., 2012, 135, 884-891.

4 J. H. Luo, Y. Xu, W. Yao, C. F. Jiang and J. G. Xu, Compos. Sci. Technol., 2015, 117, 315-321.

5 R. B. Yang, P. M. Reddy, C. J. Chang, P. A. Chen, J. K. Chen and C. C. Chang, Chem. Eng. J., 2016, 285, 497-507.

6 X. J. Zhang, G. S. Wang, W. Q. Cao, Y.-Z. Wei, J. F. Liang, L. Guo and M. S. Cao, ACS Appl. Mater. Interfaces, 2014, 6, 7471-7478.

7 T. Zhao, C. Hou, H. Zhang, R. Zhu, S. She, J. Wang, T. Li, Z. Liu and B. Wei, Sci. Rep., 2014, 4, 5619.

8 X. G. Liu, N. D. Wu, C. Y. Cui, Y. T. Li, P. P. Zhou and N. N. Bi, Mater. Lett., 2015, 149, 12-14.

9 B. Zhong, W. Liu, Y. L. Yu, L. Xia, J. L. Zhang, Z. F. Chai and G. W. Wen, Appl. Surf. Sci., 2017, 420, 858-867.

10 A. K. Sekone, Y. B. Chen, M. C. Lu, W. K. Chen, C. A. Liu and M. T. Lee, Nanoscale Res. Lett., 2016, 11, 1.

11 Y. F. Xia, Z. Qiang, B. Lee, M. L. Beckera and B. D. Vogt, CrystEngComm, 2017, 19, 4294-4303.

12 Y. Z. Zhang, S. M. Bhaway, Y. Wang, K. A. Cavicchi, M. L. Becker and B. D. Vogt, Chem. Commun., 2015, 51, 4997-5000.

13 X. Liu, B. Li, D. Geng, W. Cui, F. Yang, Z. Xie, D. Kang and Z. Zhang, Carbon, 2009, 47, 470-474. 
14 D. D. Han, S. W. Or, X. R. Dong and B. Liu, J. Alloys Compd., 2017, 695, 2605-2611.

15 B. Zhao, G. Shao, B. B. Fan, W. Y. Zhao, Y. J. Xie and R. Zhang, Phys. Chem. Chem. Phys., 2015, 17, 8802-8810.

16 S. Y. Wei, Q. Wang, J. H. Zhu, L. Y. Sun, H. F. Lin and Z. H. Guo, Nanoscale, 2011, 3, 4474-4502.

17 L. W. Jiang, Z. H. Wang, D. Li, D. Y. Geng, Y. Wang, J. An, J. He, W. Liu and Z. D. Zhang, RSC Adv., 2015, 5, 4038440392.

18 Y. J. Chen, G. Xiao, T. S. Wang, Q. Y. Ouyang, L. H. Qi, Y. Ma, P. Gao, C. L. Zhu, M. S. Cao and H. B. Jin, J. Phys. Chem. C, 2011, 115, 13603-13608.

19 Y. M. Li, D. D. Chen, X. Y. Liu, Y. C. Zhou, Q. X. Zhuang, R. L. Cai and K. Zhang, Compos. Sci. Technol., 2014, 100, 212-219.

20 S. L. Zhang, Z. W. Qi, Y. Zhao, Q. Z. Jiao, X. Ni, Y. J. Wang, Y. Chang and C. Ding, J. Alloys Compd., 2017, 694, 309-312.

21 W. Chen, X. W. Li, G. Xue, Z. Q. Wang and W. Q. Zou, Appl. Surf. Sci., 2003, 218, 215-221.

22 X. J. Zhang, S. W. Wang, G. S. Wang, Z. Li, A. P. Guo, J. Q. Zhu, D. P. Liu and P. G. Yin, $R S C A d v ., 2017$, , 22454-22460.

23 Y. Zhang, M. N. Qiu, Y. Yu, B. Y. Wen and L. L. Cheng, ACS Appl. Mater. Interfaces, 2017, 9, 809-818.

24 L. Y. Zhu, X. J. Zeng, M. Chen and R. H. Yu, RSC Adv., 2017, 7, 26801-26808.

25 F. Qin and C. Brosseau, J. Appl. Phys., 2012, 111, 061301.

26 M. X. Sui, X. L. Lv, A. M. Xie, W. D. Xu, X. H. Rong and G. J. Wu, Synth. Met., 2015, 210, 156-164.

27 M. T. Qiao, X. F. Lei, Y. Ma, L. D. Tian, K. H. Su and Q. Y. Zhang, Ind. Eng. Chem. Res., 2016, 55, 6263-6275.

28 Z. Liu, Y. Liu, S. Poyraz and X. Y. Zhang, Chem. Commun., 2011, 47, 4421-4423.

29 L. W. Jiang, Z. H. Wang, D. Y. Geng, Y. Wang, J. An, J. He, D. Li, W. Liu and Z. D. Zhang, J. Phys. Chem. C, 2016, 120, 28320-28329.

30 J. H. Luo and D. D. Gao, J. Magn. Magn. Mater., 2014, 368, 8286.

31 Y. N. Li, Y. Zhao, X. Y. Lu, Y. Zhu and L. Jiang, Nano Res., 2016, 9, 2034-2045.

32 H. C. Wang, Z. R. Yan, J. An, J. He, Y. L. Hou, H. Y. Yu, N. Ma, G. H. Yu and D. B. Sun, RSC Adv., 2016, 6, 92152-92158.

33 X. W. Wang, H. X. Yan, R. Xue and S. H. Qi, J. Mater. Sci.: Mater. Electron., 2017, 28, 519-525.

34 Y. J. Zhang, Z. M. Zhang, S. C. Xu, L. M. Yu, Y. Z. Long and Q. W. Tang, RSC Adv., 2016, 6, 6623-6630.

35 K. Zhang, M. X. Sun, W. C. Jiang, Y. Wang, D. R. Wang, F. Wu, A. M. Xie and W. Dong, RSC Adv., 2016, 6, 4305643059.

36 A. Gupta, S. Varshney, A. Goyal, P. Sambyal, B. K. Gupta and S. K. Dhawan, Mater. Lett., 2015, 158, 167-169.
37 A. Kaur, Ishpal and S. K. Dhawan, Synth. Met., 2012, 162, 1471-1477.

38 Q. S. Wang, Z. Y. Lei, Y. J. Chen, Q. Y. Ouyang, P. Gao, L. H. Qi, C. L. Zhu and J. Z. Zhang, J. Mater. Chem. A, 2013, 1, 11795-11801.

39 S. X. Xing, L. H. Tan, T. Chen, Y. H. Yang and H. Y. Chen, Chem. Commun., 2009, 45, 1653-1654.

40 P. Xu, X. Han, C. Wang, B. Zhang, X. Wang and H. L. Wang, Macromol. Rapid Commun., 2008, 29, 1392-1397.

41 X. N. Chen, J. J. Chen, F. B. Meng, L. M. Shan, M. Jiang, X. L. Xu, J. Lu, Y. Wang and Z. W. Zhou, Compos. Sci. Technol., 2016, 127, 71-78.

42 J. Ryu, Y. W. Suh, D. J. Suh and D. J. Ahn, Carbon, 2010, 48, 1990-1998.

43 P. Xu, X. Han, C. Wang, H. Zhao, J. Wang, X. Wang and B. Zhang, J. Phys. Chem. B, 2008, 112, 2775-2781.

44 N. V. Blinova, J. Stejskal, M. Trchova, J. Prokes and M. Omastova, Eur. Polym. J., 2007, 43, 2331-2341.

45 Y. Q. Shen and M. X. Wan, In situ doping polymerization of pyrrole with sulfonic acid as a dopant, Synth. Met., 1998, 96, 127-132.

46 Y. Kudoh, M. Fukuyama and S. Yoshimura, Stability study of polypyrrole and application to highly thermostable aluminum solid electrolytic capacitor, Synth. Met., 1994, 66, 157-164.

47 M. J. Antony and M. Jayakannan, J. Polym. Sci., Part B: Polym. Phys., 2009, 47, 830-846.

48 C. H. Tian, Y. C. Du, P. Xu, R. Qiang, Y. Wang, D. Ding, J. L. Xue, J. Ma, H. T. Zhao and X. J. Han, ACS Appl. Mater. Interfaces, 2015, 7, 20090-20099.

49 S. He, G. S. Wang, C. Lu, J. Liu, B. Wen, H. Liu, L. Guo and M. S. Cao, J. Mater. Chem. A, 2013, 1, 4685-4692.

50 H. L. Yu, T. S. Wang, B. Wen, M. M. Lu, Z. Xu, C. L. Zhu, Y. J. Chen, X. Y. Xue, C. W. Sun and M. S. Cao, J. Mater. Chem., 2012, 22, 21679-21685.

51 M. M. Lu, W. Q. Cao, H. L. Shi, X. Y. Fang, J. Yang, Z. L. Hou, H. B. Jin, W. Z. Wang, J. Yuan and M. S. Cao, J. Mater. Chem. A, 2014, 2, 10540-10547.

52 B. Wen, M. S. Cao, Z. L. Hou, W. L. Song, L. Zhang, M. M. Lu, H. B. Jin, X. Y. Fang, W. Z. Wang and J. Yuan, Carbon, 2013, 65, 124-139.

53 J. C. M. Garnett, Philos. Trans. R. Soc. London, 1906, 205, 237288.

54 Z. Z. Wang, H. Bi, M. Wang, P. H. Wang and X. S. Liu, Mater. Chem. Phys., 2015, 159, 173-177.

55 J. Xiang, J. Li, X. Zhang, Q. Ye, J. Xu and X. Shen, J. Mater. Chem. A, 2014, 2, 16905-16914.

56 S. L. Wen, Y. Liu, X. C. Zhao, J. W. Cheng and H. Li, Phys. Chem. Chem. Phys., 2014, 16, 18333-18340. 